Supporting information for

Explorations of Second-Order Nonlinear Optical Materials in the Alkaline Earth Barbiturates System: Noncentrosymmetric $\mathrm{Ca}\left(\mathrm{H}_{3} \mathrm{C}_{4} \mathrm{~N}_{2} \mathrm{O}_{3}\right)_{2} \bullet \mathrm{H}_{2} \mathrm{O}$ and Centrosymmetric $\mathrm{Sr}\left(\mathrm{H}_{5} \mathrm{C}_{8} \mathrm{~N}_{4} \mathrm{O}_{5}\right)_{2} \bullet 4 \mathrm{H}_{2} \mathrm{O}$

Yiyi Xu, Chensheng Lin, Donghong Lin, Min Luo,* Dan Zhao, Liling Cao and Ning Ye*

a College of Chemistry and Materials Science, Fujian Normal University, Fuzhou, Fujian 350007, P. R. China

b Key Laboratory of Optoelectronic Materials Chemistry and Physics, Fujian Institute of Research on the Structure of Matter, Chinese Academy of Sciences, Fuzhou, Fujian, 350002, P. R. China

c University of Chinese Academy of Sciences, Beijing 100049, China

d College of Chemistry and Chemical Engineering, Henan Polytechnic University, Jiaozuo, Henan 454000, China 
Table $\mathrm{S} 1$ Atomic coordinates and equivalent isotropic displacement parameters for $\mathrm{Ca}\left(\mathrm{H}_{3} \mathrm{C}_{4} \mathrm{~N}_{2} \mathrm{O}_{3}\right)_{2} \cdot \mathrm{H}_{2} \mathrm{O}$.

\begin{tabular}{lllll}
\hline Atom & $\mathrm{x}$ & $\mathrm{y}$ & $\mathrm{z}$ & $\mathrm{U}(\mathrm{eq})$ \\
\hline Ca1 & 5000 & 0 & $5626.8(11)$ & $19.6(2)$ \\
O1 & $5776.9(16)$ & $809.3(18)$ & $8496(3)$ & $32.9(6)$ \\
N3 & $6216.9(17)$ & $2480(2)$ & $9310(4)$ & $24.7(5)$ \\
C4 & $8290.6(19)$ & $1955(2)$ & $9355(4)$ & $22.2(6)$ \\
C5 & $7990.4(19)$ & $2978(2)$ & $9695(5)$ & $24.3(6)$ \\
N6 & $7497.1(17)$ & $1229(2)$ & $8925(4)$ & $24.3(5)$ \\
C7 & $6461.2(19)$ & $1478(2)$ & $8882(5)$ & $21.0(6)$ \\
C9 & $6941.0(19)$ & $3271(2)$ & $9704(5)$ & $25.0(6)$ \\
O0AA & $9214.1(13)$ & $1598.7(17)$ & $9438(4)$ & $29.4(5)$ \\
O2 & $6600.4(14)$ & $4170.5(17)$ & $10079(4)$ & $40.2(7)$ \\
O1AA & 5000 & 0 & $2082(7)$ & $102(3)$ \\
\hline
\end{tabular}

Table S2 Atomic coordinates and equivalent isotropic displacement parameters for $\mathrm{Sr}\left(\mathrm{H}_{5} \mathrm{C}_{8} \mathrm{~N}_{4} \mathrm{O}_{5}\right)_{2} \cdot 4 \mathrm{H}_{2} \mathrm{O}$

\begin{tabular}{|c|c|c|c|c|}
\hline Atom & $\mathrm{x}$ & $\mathrm{y}$ & $\mathrm{z}$ & $\mathrm{U}(\mathrm{eq})$ \\
\hline $\operatorname{Sr}(1)$ & $2404(1)$ & $6924(1)$ & 4091(1) & $47(1)$ \\
\hline $\mathrm{O}(1)$ & $6070(15)$ & $7392(6)$ & $4313(6)$ & $50(3)$ \\
\hline $\mathrm{O}(2)$ & $4770(15)$ & $5822(6)$ & $4426(6)$ & $52(3)$ \\
\hline $\mathrm{O}(3)$ & $1066(15)$ & $8053(4)$ & $4555(5)$ & $40(2)$ \\
\hline $\mathrm{O}(4)$ & $-374(16)$ & $6159(6)$ & $4364(5)$ & $55(3)$ \\
\hline $\mathrm{O}(5)$ & 2193(9) & $3624(2)$ & $9015(3)$ & $54(2)$ \\
\hline $\mathrm{O}(6)$ & 2833(9) & $5951(2)$ & $9132(3)$ & $51(1)$ \\
\hline $\mathrm{O}(7)$ & $3308(15)$ & $7896(3)$ & $7902(4)$ & $90(3)$ \\
\hline $\mathrm{O}(8)$ & $2676(9)$ & $4712(3)$ & $6735(3)$ & $52(1)$ \\
\hline $\mathrm{O}(9)$ & $2714(10)$ & $7111(3)$ & $822(3)$ & $58(2)$ \\
\hline $\mathrm{O}(10)$ & $2375(9)$ & $4758(2)$ & $660(3)$ & $51(1)$ \\
\hline $\mathrm{O}(11)$ & $2244(8)$ & $2708(2)$ & $2148(3)$ & $47(1)$ \\
\hline $\mathrm{O}(12)$ & $2135(9)$ & $4059(3)$ & 4294(3) & $58(2)$ \\
\hline $\mathrm{O}(13)$ & $2280(9)$ & $5962(2)$ & $3055(3)$ & $47(1)$ \\
\hline $\mathrm{O}(14)$ & $2949(10)$ & $6828(3)$ & $5555(3)$ & $62(2)$ \\
\hline $\mathrm{N}(1)$ & 2501(9) & $4196(3)$ & 7884(3) & $41(1)$ \\
\hline $\mathrm{N}(2)$ & $2579(9)$ & $4790(3)$ & $9038(3)$ & $39(1)$ \\
\hline $\mathrm{N}(3)$ & $2925(12)$ & $6705(3)$ & $7851(4)$ & $55(2)$ \\
\hline $\mathrm{N}(4)$ & $3063(10)$ & $7349(3)$ & $6722(4)$ & $47(2)$ \\
\hline $\mathrm{N}(5)$ & $2118(9)$ & 3393(3) & $3208(3)$ & $42(1)$ \\
\hline $\mathrm{N}(6)$ & 2211(9) & $4593(3)$ & $3123(4)$ & $42(1)$ \\
\hline $\mathrm{N}(7)$ & $2539(11)$ & $6494(3)$ & $1920(4)$ & $46(2)$ \\
\hline $\mathrm{N}(8)$ & $2561(10)$ & $5927(3)$ & 763(4) & $44(2)$ \\
\hline$C(1)$ & $2408(11)$ & $4173(3)$ & $8659(4)$ & $41(2)$ \\
\hline$C(2)$ & $2735(11)$ & $5438(3)$ & $8686(4)$ & $38(1)$ \\
\hline$C(3)$ & $2737(10)$ & $5443(3)$ & $7867(4)$ & $35(1)$ \\
\hline$C(4)$ & $2651(10)$ & 4801(3) & $7454(4)$ & $36(1)$ \\
\hline$C(5)$ & $2840(10)$ & $6096(3)$ & $7441(5)$ & $40(2)$ \\
\hline$C(6)$ & $2858(10)$ & $6133(3)$ & $6663(4)$ & $38(2)$ \\
\hline$C(7)$ & $2983(12)$ & $6767(4)$ & $6259(5)$ & $44(2)$ \\
\hline$C(8)$ & $3106(15)$ & $7348(4)$ & $7526(4)$ & $55(2)$ \\
\hline
\end{tabular}




\begin{tabular}{lllll}
$\mathrm{C}(9)$ & $2159(10)$ & $4015(3)$ & $3596(4)$ & $38(2)$ \\
$\mathrm{C}(10)$ & $2209(11)$ & $3312(3)$ & $2418(4)$ & $39(2)$ \\
$\mathrm{C}(11)$ & $2194(10)$ & $3932(3)$ & $1973(4)$ & $37(1)$ \\
$\mathrm{C}(12)$ & $2222(10)$ & $4575(3)$ & $2335(4)$ & $36(1)$ \\
$\mathrm{C}(13)$ & $2319(10)$ & $5248(3)$ & $1922(4)$ & $36(1)$ \\
$\mathrm{C}(14)$ & $2355(11)$ & $5885(3)$ & $2350(4)$ & $37(2)$ \\
$\mathrm{C}(15)$ & $2419(10)$ & $5266(3)$ & $1101(4)$ & $39(2)$ \\
$\mathrm{C}(16)$ & $2620(12)$ & $6547(3)$ & $1147(4)$ & $44(2)$ \\
\hline
\end{tabular}

Table S3 Bond lengths $(\AA)$ for $\mathrm{Ca}\left(\mathrm{H}_{3} \mathrm{C}_{4} \mathrm{~N}_{2} \mathrm{O}_{3}\right)_{2} \cdot \mathrm{H}_{2} \mathrm{O}$.

\begin{tabular}{llll}
\hline Ca1-O1\#1 & $2.426(3)$ & N3-C7 & $1.341(5)$ \\
Ca1-O1 & $2.426(3)$ & N3-C9 & $1.391(4)$ \\
Ca1-O0AA\#2 & $2.404(4)$ & C4-C5 & $1.373(5)$ \\
Ca1-O0AA\#3 & $2.404(4)$ & C4-N6 & $1.400(4)$ \\
Ca1-O2\#4 & $2.330(3)$ & C4-O0AA & $1.265(3)$ \\
Ca1-O2\#5 & $2.330(3)$ & C5-C9 & $1.391(4)$ \\
Ca1-O1AA & $2.423(6)$ & N6-C7 & $1.361(4)$ \\
O1-C7 & $1.246(4)$ & C9-O2 & $1.247(4)$ \\
\hline
\end{tabular}

\#1 1-X,-Y,+Z; \#2 3/2-X,+Y,-1/2+Z; \#3 -1/2+X,-Y,-1/2+Z;

\#4 1-X,1/2-Y,-1/2+Z; \#5 +X,-1/2+Y,-1/2+Z

Table S4 Bond lengths $(\AA)$ for $\operatorname{Sr}\left(\mathrm{H}_{5} \mathrm{C}_{8} \mathrm{~N}_{4} \mathrm{O}_{5}\right)_{2} \cdot 4 \mathrm{H}_{2} \mathrm{O}$.

\begin{tabular}{llll}
\hline $\mathrm{Sr}(1)-\mathrm{O}(1)$ & $2.605(10)$ & $\mathrm{N}(2)-\mathrm{C}(1)$ & $1.350(9)$ \\
$\mathrm{Sr}(1)-\mathrm{O}(2)$ & $2.660(10)$ & $\mathrm{N}(2)-\mathrm{C}(2)$ & $1.391(8)$ \\
$\mathrm{Sr}(1)-\mathrm{O}(3)$ & $2.514(7)$ & $\mathrm{N}(3)-\mathrm{H}(3)$ & 0.8600 \\
$\mathrm{Sr}(1)-\mathrm{O}(4)$ & $2.471(8)$ & $\mathrm{N}(3)-\mathrm{C}(5)$ & $1.361(9)$ \\
$\mathrm{Sr}(1)-\mathrm{O}(7) \# 1$ & $3.225(9)$ & $\mathrm{N}(3)-\mathrm{C}(8)$ & $1.367(9)$ \\
$\mathrm{Sr}(1)-\mathrm{O}(11) \# 2$ & $2.651(5)$ & $\mathrm{N}(4)-\mathrm{H}(4)$ & 0.8600 \\
$\mathrm{Sr}(1)-\mathrm{O}(13)$ & $2.560(5)$ & $\mathrm{N}(4)-\mathrm{C}(7)$ & $1.369(9)$ \\
$\mathrm{Sr}(1)-\mathrm{O}(14)$ & $2.510(6)$ & $\mathrm{N}(4)-\mathrm{C}(8)$ & $1.384(9)$ \\
$\mathrm{O}(1)-\mathrm{H}(1 \mathrm{~A})$ & 0.8858 & $\mathrm{~N}(5)-\mathrm{H}(5)$ & 0.8600 \\
$\mathrm{O}(1)-\mathrm{H}(1 \mathrm{~B})$ & 0.8858 & $\mathrm{~N}(5)-\mathrm{C}(9)$ & $1.365(9)$ \\
$\mathrm{O}(2)-\mathrm{H}(2 \mathrm{~A})$ & 0.8898 & $\mathrm{~N}(5)-\mathrm{C}(10)$ & $1.382(9)$ \\
$\mathrm{O}(2)-\mathrm{H}(2 \mathrm{~B})$ & 0.8895 & $\mathrm{~N}(6)-\mathrm{H}(6)$ & 0.8600 \\
$\mathrm{O}(3)-\mathrm{H}(3 \mathrm{~A})$ & 0.9432 & $\mathrm{~N}(6)-\mathrm{C}(9)$ & $1.378(9)$ \\
$\mathrm{O}(3)-\mathrm{H}(3 \mathrm{~B})$ & 0.9397 & $\mathrm{~N}(6)-\mathrm{C}(12)$ & $1.361(9)$ \\
$\mathrm{O}(4)-\mathrm{H}(4 \mathrm{~A})$ & 1.0157 & $\mathrm{~N}(7)-\mathrm{H}(7)$ & 0.8600 \\
$\mathrm{O}(4)-\mathrm{H}(4 \mathrm{~B})$ & 1.0219 & $\mathrm{~N}(7)-\mathrm{C}(14)$ & $1.398(8)$ \\
$\mathrm{O}(5)-\mathrm{C}(1)$ & $1.236(8)$ & $\mathrm{N}(7)-\mathrm{C}(16)$ & $1.347(9)$ \\
$\mathrm{O}(6)-\mathrm{C}(2)$ & $1.244(8)$ & $\mathrm{N}(8)-\mathrm{H}(8)$ & 0.8600 \\
$\mathrm{O}(7)-\mathrm{Sr}(1) \# 3$ & $3.225(9)$ & $\mathrm{N}(8)-\mathrm{C}(15)$ & $1.404(9)$ \\
$\mathrm{O}(7)-\mathrm{C}(8)$ & $1.232(9)$ & $\mathrm{N}(8)-\mathrm{C}(16)$ & $1.358(8)$ \\
$\mathrm{O}(8)-\mathrm{C}(4)$ & $1.254(8)$ & $\mathrm{C}(2)-\mathrm{C}(3)$ & $1.414(10)$ \\
$\mathrm{O}(9)-\mathrm{C}(16)$ & $1.223(9)$ & $\mathrm{C}(3)-\mathrm{C}(4)$ & $1.420(9)$ \\
$\mathrm{O}(10)-\mathrm{C}(15)$ & $1.232(8)$ & $\mathrm{C}(3)-\mathrm{C}(5)$ & $1.456(9)$ \\
$\mathrm{O}(11)-\mathrm{Sr}(1) \# 4$ & $2.651(5)$ & $\mathrm{C}(5)-\mathrm{C}(6)$ & $1.347(11)$ \\
$\mathrm{O}(11)-\mathrm{C}(10)$ & $1.249(8)$ & $\mathrm{C}(6)-\mathrm{H}(6 \mathrm{~A})$ & 0.9300 \\
$\mathrm{O}(12)-\mathrm{C}(9)$ & $1.211(9)$ & $\mathrm{C}(6)-\mathrm{C}(7)$ & $1.409(9)$ \\
& & & \\
& & &
\end{tabular}




\begin{tabular}{llll}
$\mathrm{O}(13)-\mathrm{C}(14)$ & $1.234(8)$ & $\mathrm{C}(10)-\mathrm{C}(11)$ & $1.412(9)$ \\
$\mathrm{O}(14)-\mathrm{C}(7)$ & $1.216(10)$ & $\mathrm{C}(11)-\mathrm{H}(11)$ & 0.9300 \\
$\mathrm{~N}(1)-\mathrm{H}(1)$ & 0.8600 & $\mathrm{C}(11)-\mathrm{C}(12)$ & $1.380(9)$ \\
$\mathrm{N}(1)-\mathrm{C}(1)$ & $1.347(9)$ & $\mathrm{C}(12)-\mathrm{C}(13)$ & $1.478(9)$ \\
$\mathrm{N}(1)-\mathrm{C}(4)$ & $1.387(9)$ & $\mathrm{C}(13)-\mathrm{C}(14)$ & $1.425(9)$ \\
$\mathrm{N}(2)-\mathrm{H}(2)$ & 0.8600 & $\mathrm{C}(13)-\mathrm{C}(15)$ & $1.427(10)$ \\
\hline
\end{tabular}

$\# 1 \mathrm{x}-1 / 2,-\mathrm{y}+3 / 2, \mathrm{z}-1 / 2 ; \quad \# 2-\mathrm{x}+1 / 2, \mathrm{y}+1 / 2,-\mathrm{z}+1 / 2$

$\# 3 \mathrm{x}+1 / 2,-\mathrm{y}+3 / 2, \mathrm{z}+1 / 2 ; \# 4-\mathrm{x}+1 / 2, \mathrm{y}-1 / 2,-\mathrm{z}+1 / 2$

Table S5 Anisotropic displacement parameters $\left(\AA^{2} \times 10^{3}\right)$ for $\mathrm{Ca}\left(\mathrm{H}_{3} \mathrm{C}_{4} \mathrm{~N}_{2} \mathrm{O}_{3}\right)_{2} \cdot \mathrm{H}_{2} \mathrm{O}$.

The anisotropic displacement factor exponent takes the form: $-2 \pi^{2}\left[\mathrm{~h}^{2} \mathrm{a}^{* 2} \mathrm{U} 11+\ldots+2 \mathrm{hka} *\right.$ $b^{*} \mathrm{U} 12$ ].

\begin{tabular}{lllllll}
\hline Atom & $\mathrm{U} 11$ & $\mathrm{U} 22$ & $\mathrm{U} 33$ & $\mathrm{U} 12$ & $\mathrm{U} 13$ & $\mathrm{U} 23$ \\
\hline Ca1 & $14.0(3)$ & $13.8(4)$ & $31.1(4)$ & $0.2(2)$ & 0 & 0 \\
O1 & $28.7(11)$ & $25.0(13)$ & $44.9(14)$ & $-9.7(8)$ & $-0.1(10)$ & $-4.6(11)$ \\
N3 & $13.3(9)$ & $19.5(12)$ & $41.3(15)$ & $-0.7(9)$ & $-0.6(9)$ & $-1.8(12)$ \\
C4 & $17.4(11)$ & $21.8(15)$ & $27.4(16)$ & $-0.3(10)$ & $-0.1(11)$ & $1.6(13)$ \\
C5 & $16.2(11)$ & $17.7(14)$ & $38.9(17)$ & $1.0(10)$ & $-3.2(12)$ & $-4.5(14)$ \\
N6 & $21.8(10)$ & $13.3(12)$ & $37.9(14)$ & $1.0(9)$ & $0.9(11)$ & $-2.0(11)$ \\
C7 & $18.8(11)$ & $19.0(15)$ & $25.2(14)$ & $-4.4(9)$ & $2.2(11)$ & $-2.4(12)$ \\
C9 & $19.3(12)$ & $16.9(14)$ & $38.8(16)$ & $0.7(10)$ & $-2.8(13)$ & $-2.4(13)$ \\
O0AA & $14.9(8)$ & $26.2(12)$ & $47.2(15)$ & $4.8(8)$ & $-0.1(9)$ & $3.5(11)$ \\
O2 & $22.4(9)$ & $17.8(12)$ & $80(2)$ & $4.4(7)$ & $2.2(10)$ & $-10.6(12)$ \\
O1AA & $158(6)$ & $113(6)$ & $33(3)$ & $-98(5)$ & 0 & 0 \\
\hline
\end{tabular}

Table S6 Anisotropic displacement parameters $(\AA 2 \times 103)$ for $\operatorname{Sr}\left(\mathrm{H}_{5} \mathrm{C}_{8} \mathrm{~N}_{4} \mathrm{O}_{5}\right)_{2} \cdot 4 \mathrm{H}_{2} \mathrm{O}$.

The anisotropic displacement factor exponent takes the form: $-2 \pi 2[\mathrm{~h} 2 \mathrm{a} * 2 \mathrm{U} 11+\ldots+$ $2 \mathrm{hka}{ }^{*} \mathrm{~b} * \mathrm{U} 12$ ].

\begin{tabular}{lllllll}
\hline Atom & $\mathrm{U} 11$ & $\mathrm{U} 22$ & $\mathrm{U} 33$ & $\mathrm{U} 12$ & $\mathrm{U} 13$ & $\mathrm{U} 23$ \\
\hline $\mathrm{Sr}(1)$ & $77(1)$ & $27(1)$ & $40(1)$ & $-3(1)$ & $14(1)$ & $-4(1)$ \\
$\mathrm{O}(1)$ & $42(6)$ & $71(7)$ & $35(5)$ & $-5(5)$ & $1(4)$ & $-8(5)$ \\
$\mathrm{O}(2)$ & $45(6)$ & $66(7)$ & $41(6)$ & $-17(5)$ & $-9(4)$ & $23(5)$ \\
$\mathrm{O}(3)$ & $57(6)$ & $30(5)$ & $31(5)$ & $-11(3)$ & $-7(4)$ & $27(4)$ \\
$\mathrm{O}(4)$ & $62(6)$ & $91(8)$ & $14(4)$ & $-16(4)$ & $13(4)$ & $-56(6)$ \\
$\mathrm{O}(5)$ & $97(4)$ & $19(2)$ & $45(3)$ & $0(2)$ & $12(3)$ & $-10(2)$ \\
$\mathrm{O}(6)$ & $85(4)$ & $21(2)$ & $46(3)$ & $-5(2)$ & $8(3)$ & $-6(2)$ \\
$\mathrm{O}(7)$ & $184(9)$ & $33(3)$ & $58(4)$ & $-10(3)$ & $32(5)$ & $8(4)$ \\
$\mathrm{O}(8)$ & $69(4)$ & $43(3)$ & $45(3)$ & $-1(2)$ & $15(3)$ & $-8(3)$ \\
$\mathrm{O}(9)$ & $104(5)$ & $24(2)$ & $47(3)$ & $6(2)$ & $23(3)$ & $2(3)$ \\
$\mathrm{O}(10)$ & $85(4)$ & $25(2)$ & $44(3)$ & $-6(2)$ & $13(3)$ & $-4(2)$ \\
$\mathrm{O}(11)$ & $76(4)$ & $19(2)$ & $45(3)$ & $-4(2)$ & $9(2)$ & $2(2)$ \\
$\mathrm{O}(12)$ & $74(4)$ & $59(4)$ & $40(3)$ & $-4(3)$ & $8(3)$ & $5(3)$ \\
$\mathrm{O}(13)$ & $79(4)$ & $25(2)$ & $38(3)$ & $-3(2)$ & $11(2)$ & $3(2)$ \\
$\mathrm{O}(14)$ & $83(4)$ & $65(4)$ & $38(3)$ & $-5(3)$ & $12(3)$ & $4(3)$ \\
$\mathrm{N}(1)$ & $58(4)$ & $21(3)$ & $45(3)$ & $-4(2)$ & $11(3)$ & $-4(2)$ \\
$\mathrm{N}(2)$ & $58(4)$ & $24(3)$ & $34(3)$ & $0(2)$ & $7(3)$ & $-4(2)$ \\
$\mathrm{N}(3)$ & $86(5)$ & $30(3)$ & $48(4)$ & $6(3)$ & $9(3)$ & $5(3)$ \\
& & & & $\mathrm{S} 4$ & &
\end{tabular}




\begin{tabular}{lllllll}
$\mathrm{N}(4)$ & $77(4)$ & $21(3)$ & $44(3)$ & $6(2)$ & $10(3)$ & $0(3)$ \\
$\mathrm{N}(5)$ & $57(4)$ & $27(3)$ & $40(3)$ & $4(2)$ & $6(3)$ & $-1(2)$ \\
$\mathrm{N}(6)$ & $53(4)$ & $24(3)$ & $49(4)$ & $2(2)$ & $10(3)$ & $2(2)$ \\
$\mathrm{N}(7)$ & $77(4)$ & $20(3)$ & $40(3)$ & $-2(2)$ & $10(3)$ & $-2(3)$ \\
$\mathrm{N}(8)$ & $73(4)$ & $23(3)$ & $39(3)$ & $-3(2)$ & $17(3)$ & $2(3)$ \\
$\mathrm{C}(1)$ & $47(4)$ & $30(3)$ & $46(4)$ & $-3(3)$ & $4(3)$ & $-5(3)$ \\
$\mathrm{C}(2)$ & $47(4)$ & $24(3)$ & $42(4)$ & $0(3)$ & $1(3)$ & $-3(3)$ \\
$\mathrm{C}(3)$ & $35(3)$ & $22(3)$ & $49(4)$ & $3(3)$ & $5(3)$ & $-3(2)$ \\
$\mathrm{C}(4)$ & $37(3)$ & $33(3)$ & $40(4)$ & $-2(3)$ & $6(3)$ & $-3(3)$ \\
$\mathrm{C}(5)$ & $31(3)$ & $24(3)$ & $67(5)$ & $9(3)$ & $7(3)$ & $1(2)$ \\
$\mathrm{C}(6)$ & $43(4)$ & $18(3)$ & $53(4)$ & $7(3)$ & $8(3)$ & $1(2)$ \\
$\mathrm{C}(7)$ & $54(4)$ & $33(3)$ & $45(4)$ & $2(3)$ & $4(3)$ & $2(3)$ \\
$\mathrm{C}(8)$ & $98(7)$ & $26(3)$ & $41(4)$ & $3(3)$ & $14(4)$ & $6(4)$ \\
$\mathrm{C}(9)$ & $41(4)$ & $26(3)$ & $47(4)$ & $-1(3)$ & $9(3)$ & $1(3)$ \\
$\mathrm{C}(10)$ & $50(4)$ & $25(3)$ & $42(4)$ & $1(3)$ & $3(3)$ & $-3(3)$ \\
$\mathrm{C}(11)$ & $48(4)$ & $25(3)$ & $38(4)$ & $1(3)$ & $6(3)$ & $5(3)$ \\
$\mathrm{C}(12)$ & $35(3)$ & $27(3)$ & $47(4)$ & $0(3)$ & $7(3)$ & $3(2)$ \\
$\mathrm{C}(13)$ & $44(4)$ & $20(3)$ & $43(4)$ & $-2(2)$ & $4(3)$ & $3(2)$ \\
$\mathrm{C}(14)$ & $48(4)$ & $23(3)$ & $41(4)$ & $2(3)$ & $7(3)$ & $-2(3)$ \\
$\mathrm{C}(15)$ & $48(4)$ & $22(3)$ & $47(4)$ & $-8(3)$ & $9(3)$ & $3(3)$ \\
$\mathrm{C}(16)$ & $64(5)$ & $25(3)$ & $44(4)$ & $-4(3)$ & $14(3)$ & $1(3)$ \\
\hline
\end{tabular}

Table S7. Geometrical Factors (g) at $1064 \mathrm{~nm}$ for $\mathrm{Ca}\left(\mathrm{H}_{3} \mathrm{C}_{4} \mathrm{~N}_{2} \mathrm{O}_{3}\right)_{2} \cdot \mathrm{H}_{2} \mathrm{O}$.

\begin{tabular}{lll}
\hline $\mathrm{g}_{311} / \mathrm{N}$ & $\mathrm{g}_{322} / \mathrm{N}$ & $\mathrm{g}_{333} / \mathrm{N}$ \\
\hline-0.061 & 0.084 & -0.073 \\
\hline
\end{tabular}




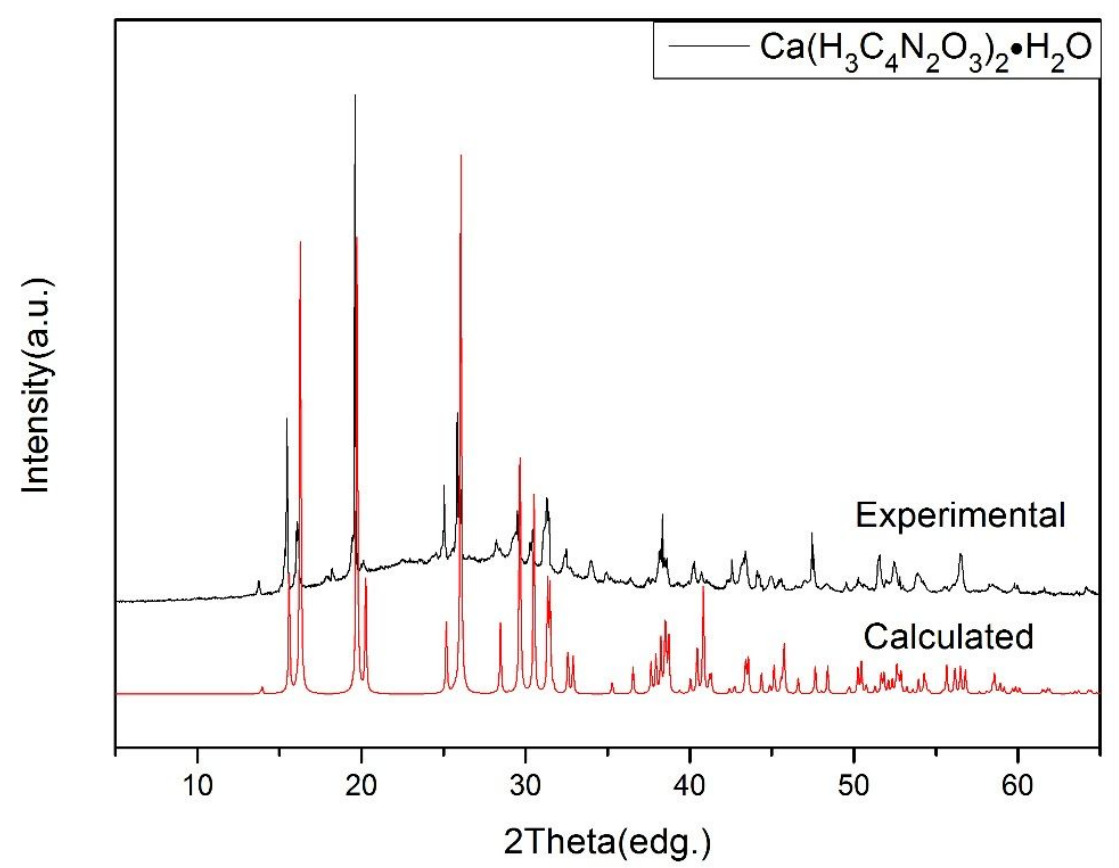

Figure S1. Experimental and calculated XRD patterns for $\mathrm{Ca}\left(\mathrm{H}_{3} \mathrm{C}_{4} \mathrm{~N}_{2} \mathrm{O}_{3}\right)_{2} \cdot \mathrm{H}_{2} \mathrm{O}$. The black curves are the patterns of samples, the red are the calculated ones.

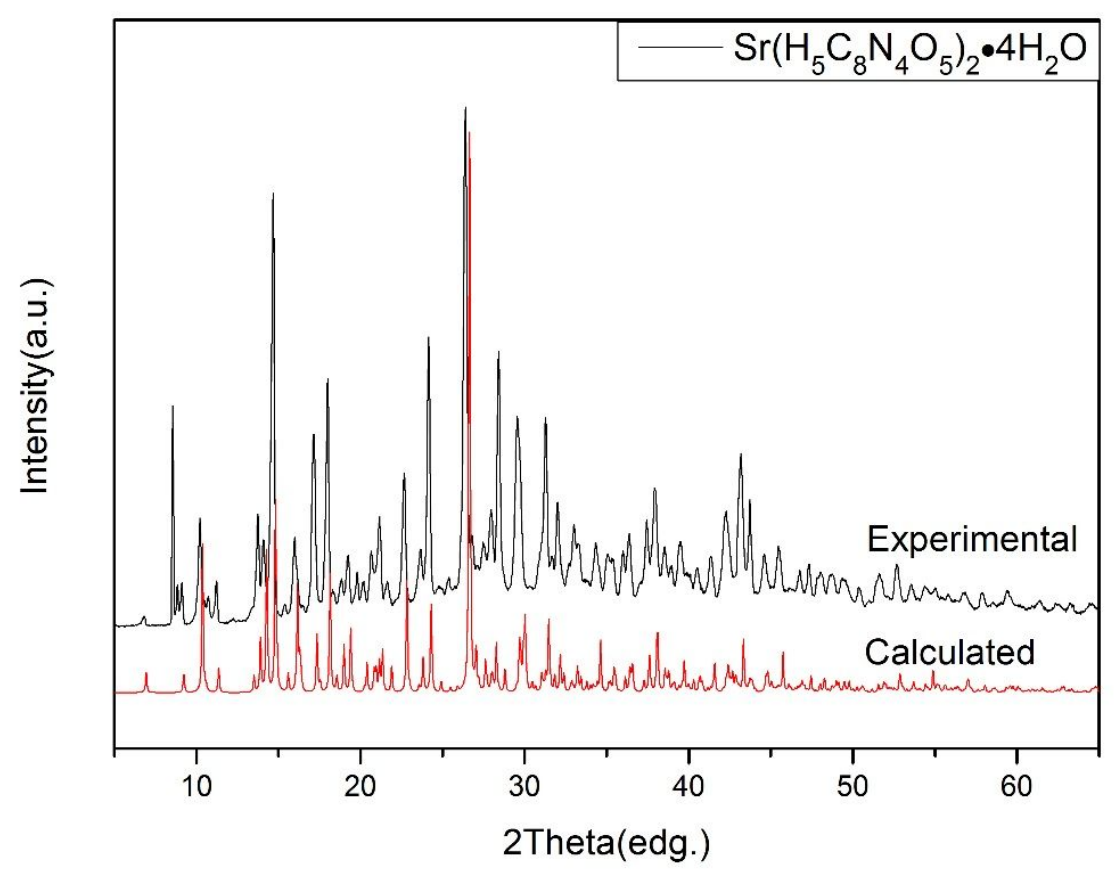

Figure S2. Experimental and calculated XRD patterns for $\mathrm{Sr}\left(\mathrm{H}_{5} \mathrm{C}_{8} \mathrm{~N}_{4} \mathrm{O}_{5}\right)_{2} \cdot 4 \mathrm{H}_{2} \mathrm{O}$. The black curves are the patterns of samples, the red are the calculated ones. 


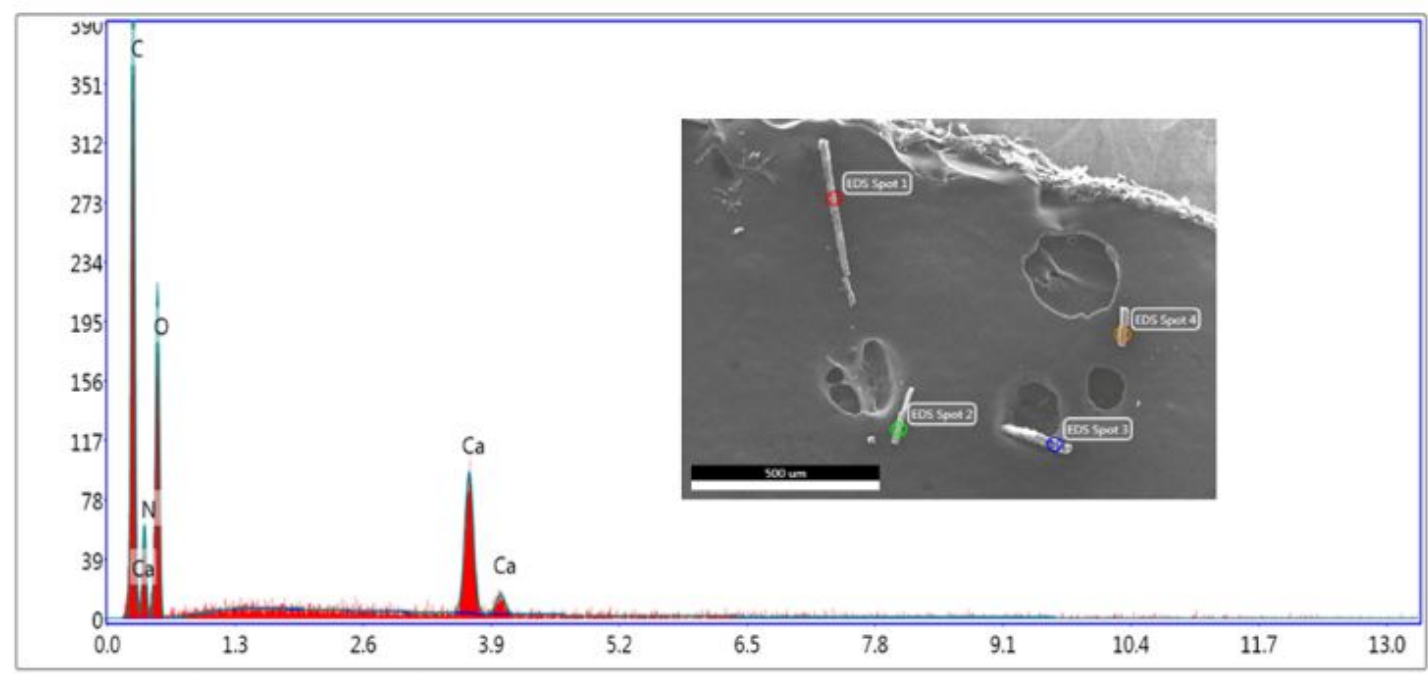

Lsec: 9.50 Cnts 0.000 keV Det: Octane Plus Det

Figure S3. Energy dispersive X-ray spectroscopy analysis of $\mathrm{Ca}\left(\mathrm{H}_{3} \mathrm{C}_{4} \mathrm{~N}_{2} \mathrm{O}_{3}\right)_{2} \cdot \mathrm{H}_{2} \mathrm{O}$.

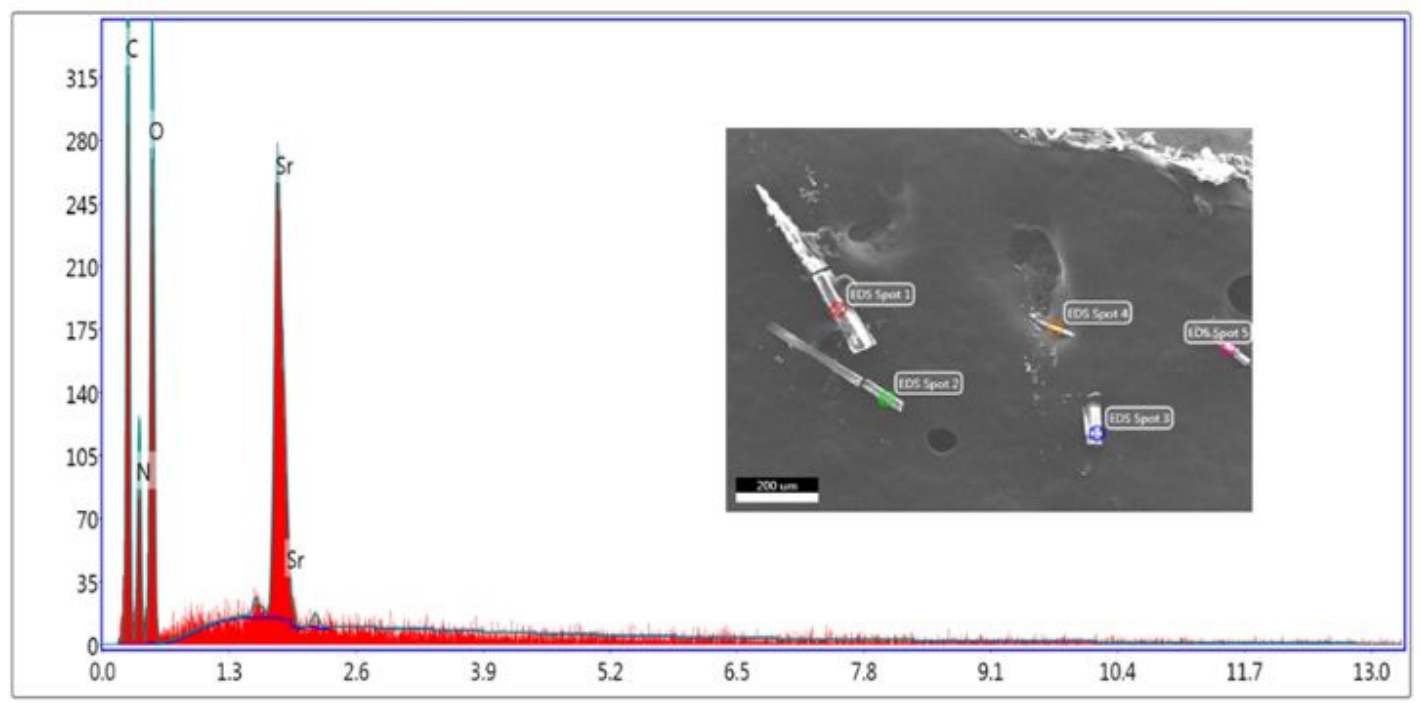

Lsec: 13.50 Cnts 0.000 keV Det: Octane Plus Det

Figure $\mathrm{S} 4$. Energy dispersive X-ray spectroscopy analysis of $\operatorname{Sr}\left(\mathrm{H}_{5} \mathrm{C}_{8} \mathrm{~N}_{4} \mathrm{O}_{5}\right)_{2} \cdot 4 \mathrm{H}_{2} \mathrm{O}$. 

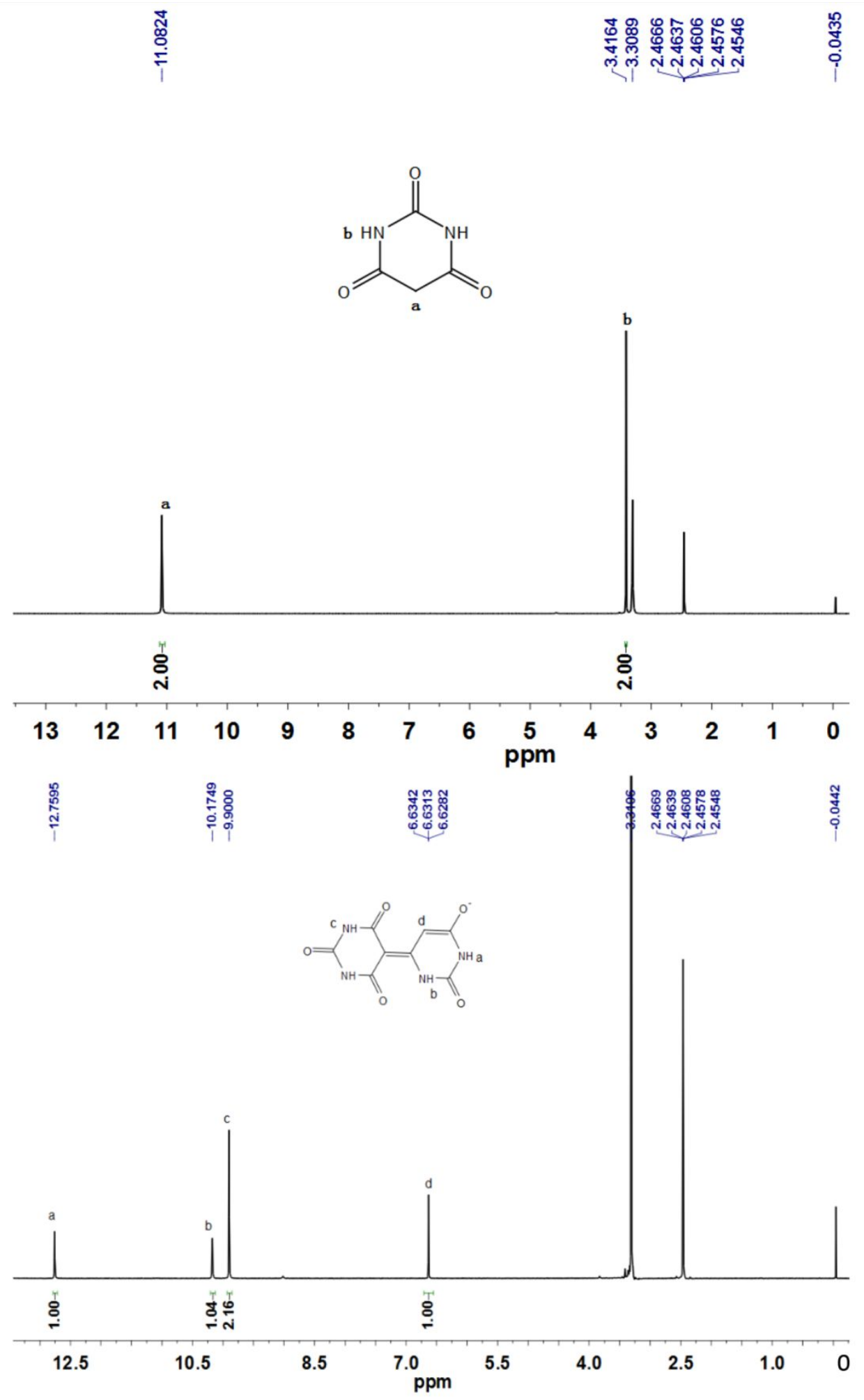

Figure S5. Nuclear magnetic resonance(NMR) spectroscopy of (a) ingredient $\left(\mathrm{H}_{4} \mathrm{C}_{4} \mathrm{~N}_{2} \mathrm{O}_{3}\right)$ and (b) crystal $\mathrm{Sr}\left(\mathrm{H}_{5} \mathrm{C}_{8} \mathrm{~N}_{4} \mathrm{O}_{5}\right)_{2} \cdot 4 \mathrm{H}_{2} \mathrm{O}$.

a: ${ }^{1} \mathrm{H}$ NMR (DMSO-d $\left.6,600 \mathrm{MHz}\right): \delta / \mathrm{ppm}=11.08(\mathrm{~s}, 2 \mathrm{H}), 3.42(\mathrm{~s}, 2 \mathrm{H})$.

$\mathrm{b}:{ }^{1} \mathrm{H}$ NMR (DMSO-d 6 , $\left.600 \mathrm{MHz}\right): \delta / \mathrm{ppm}=12.76(\mathrm{~s}, 1 \mathrm{H}), 10,17(\mathrm{~s}, 1 \mathrm{H}), 9.90(\mathrm{~s}, 2 \mathrm{H})$, $6.63(\mathrm{~s}, 1 \mathrm{H})$. 


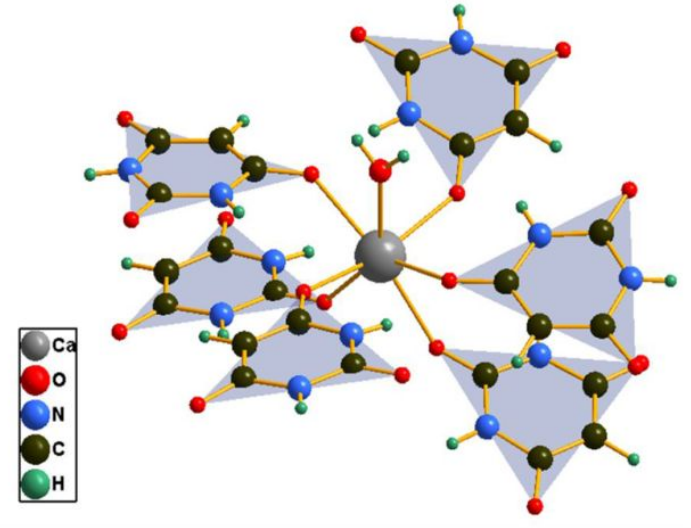

$\mathrm{Ca}\left(\mathrm{H}_{3} \mathrm{C}_{4} \mathrm{~N}_{2} \mathrm{O}_{3}\right)_{2} \bullet \mathrm{H}_{2} \mathrm{O}$

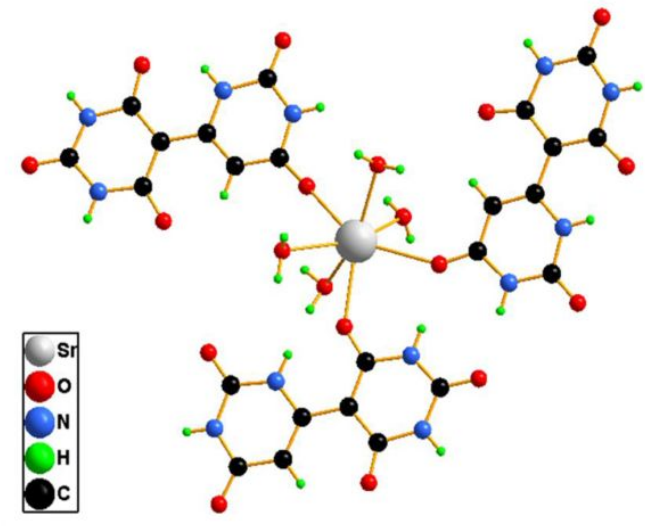

$\mathrm{Sr}\left(\mathrm{H}_{5} \mathrm{C}_{8} \mathrm{~N}_{4} \mathrm{O}_{5}\right)_{2} \bullet 4 \mathrm{H}_{2} \mathrm{O}$

Figure S6. Coordination environment of metal cations in $\mathrm{Ca}\left(\mathrm{H}_{3} \mathrm{C}_{4} \mathrm{~N}_{2} \mathrm{O}_{3}\right)_{2} \cdot \mathrm{H}_{2} \mathrm{O}$ and $\mathrm{Sr}\left(\mathrm{H}_{5} \mathrm{C}_{8} \mathrm{~N}_{4} \mathrm{O}_{5}\right)_{2} \cdot 4 \mathrm{H}_{2} \mathrm{O}$.

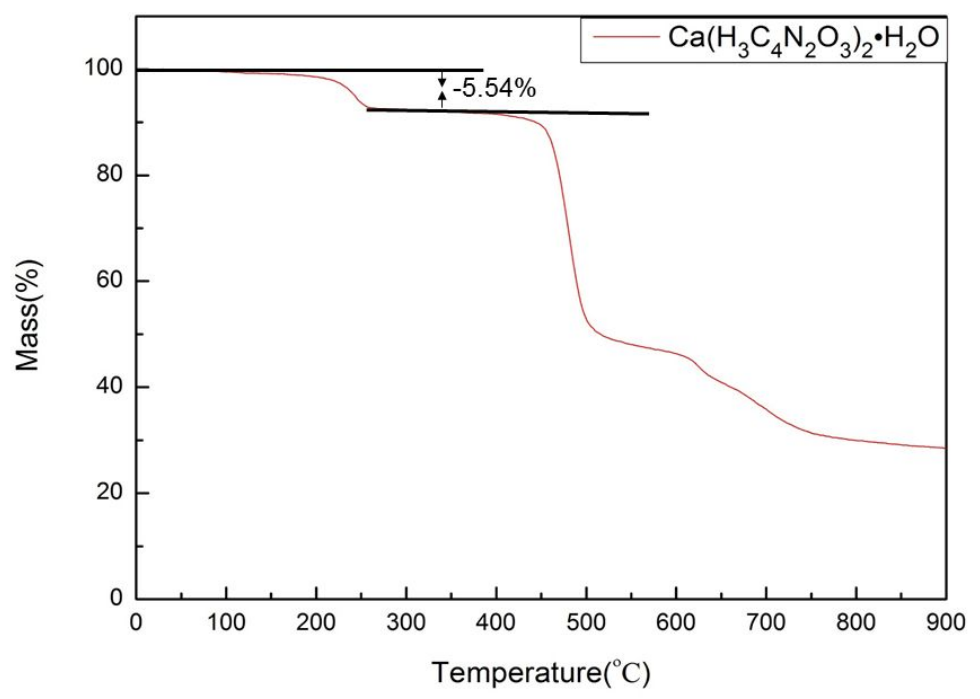

Figure S7. TG diagrams for $\mathrm{Ca}\left(\mathrm{H}_{3} \mathrm{C}_{4} \mathrm{~N}_{2} \mathrm{O}_{3}\right)_{2} \cdot \mathrm{H}_{2} \mathrm{O}$. 


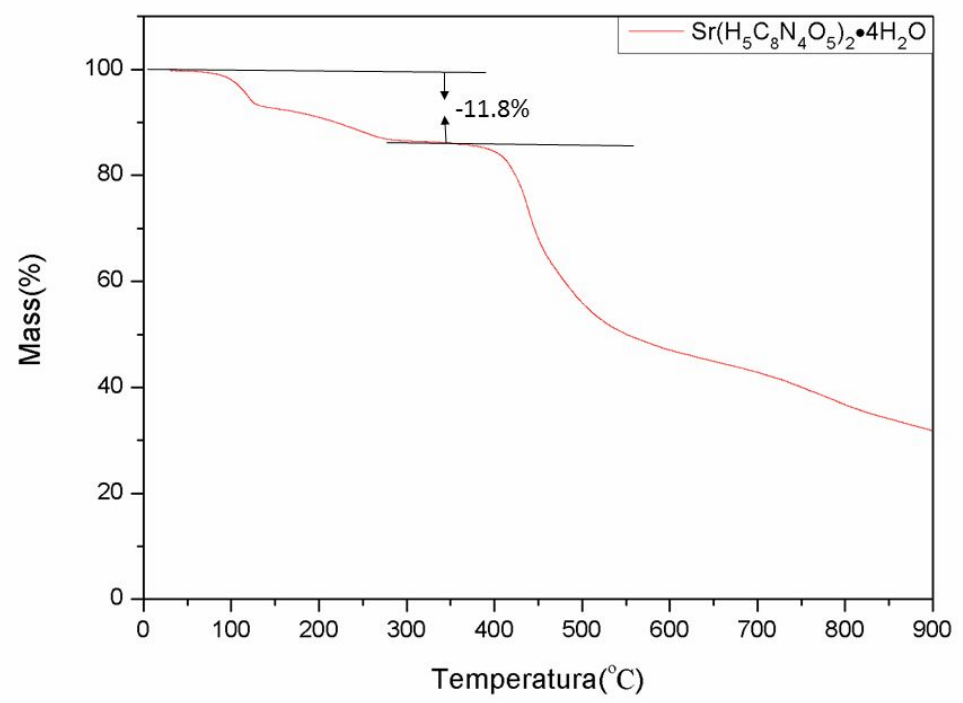

Figure S8. TG diagrams for $\operatorname{Sr}\left(\mathrm{H}_{5} \mathrm{C}_{8} \mathrm{~N}_{4} \mathrm{O}_{5}\right)_{2} \cdot 4 \mathrm{H}_{2} \mathrm{O}$.

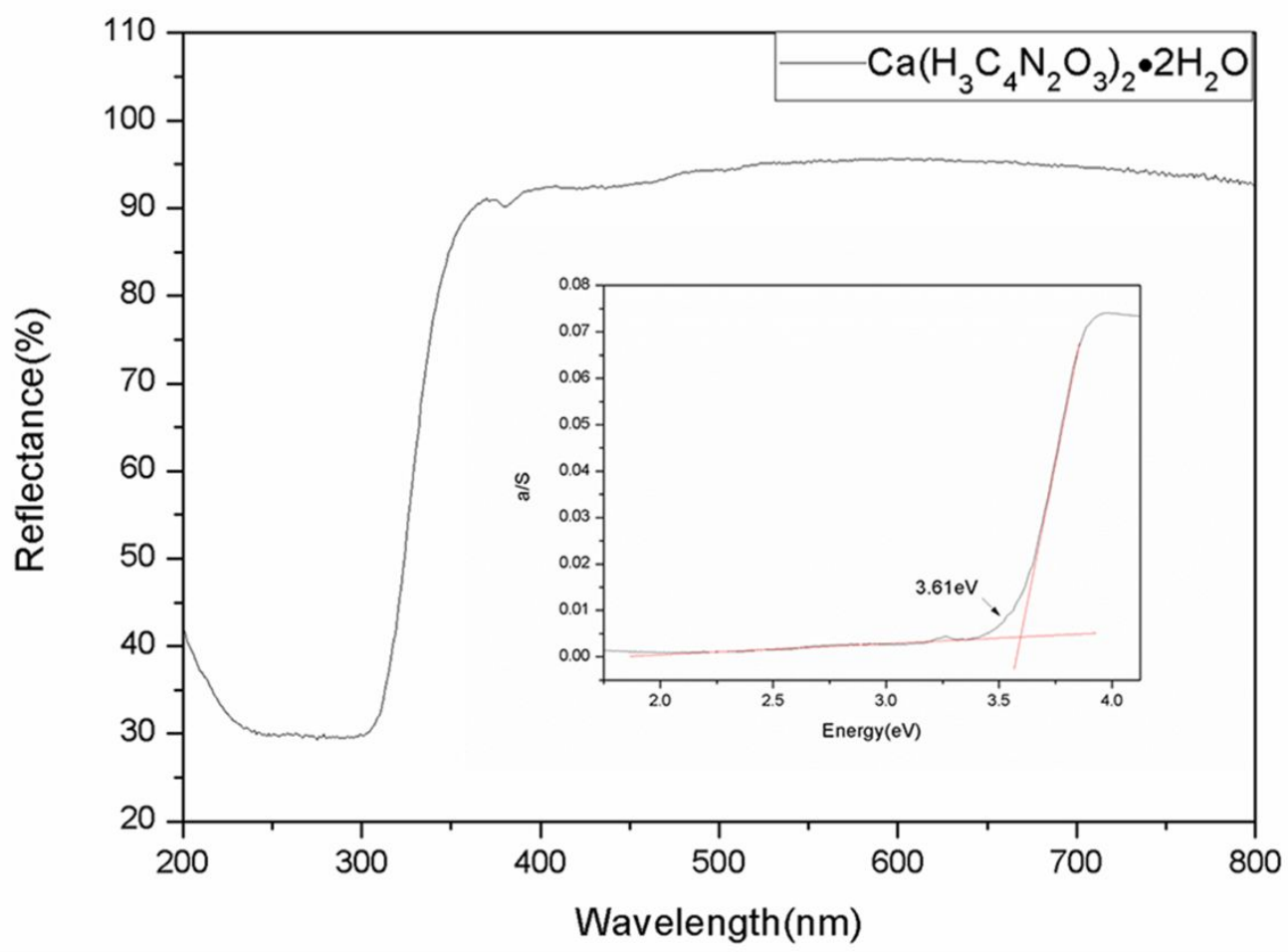

Figure S9. UV-vis-NIR diffuse reflectance spectrum of $\mathrm{Ca}\left(\mathrm{H}_{3} \mathrm{C}_{4} \mathrm{~N}_{2} \mathrm{O}_{3}\right)_{2} \cdot \mathrm{H}_{2} \mathrm{O}$. 


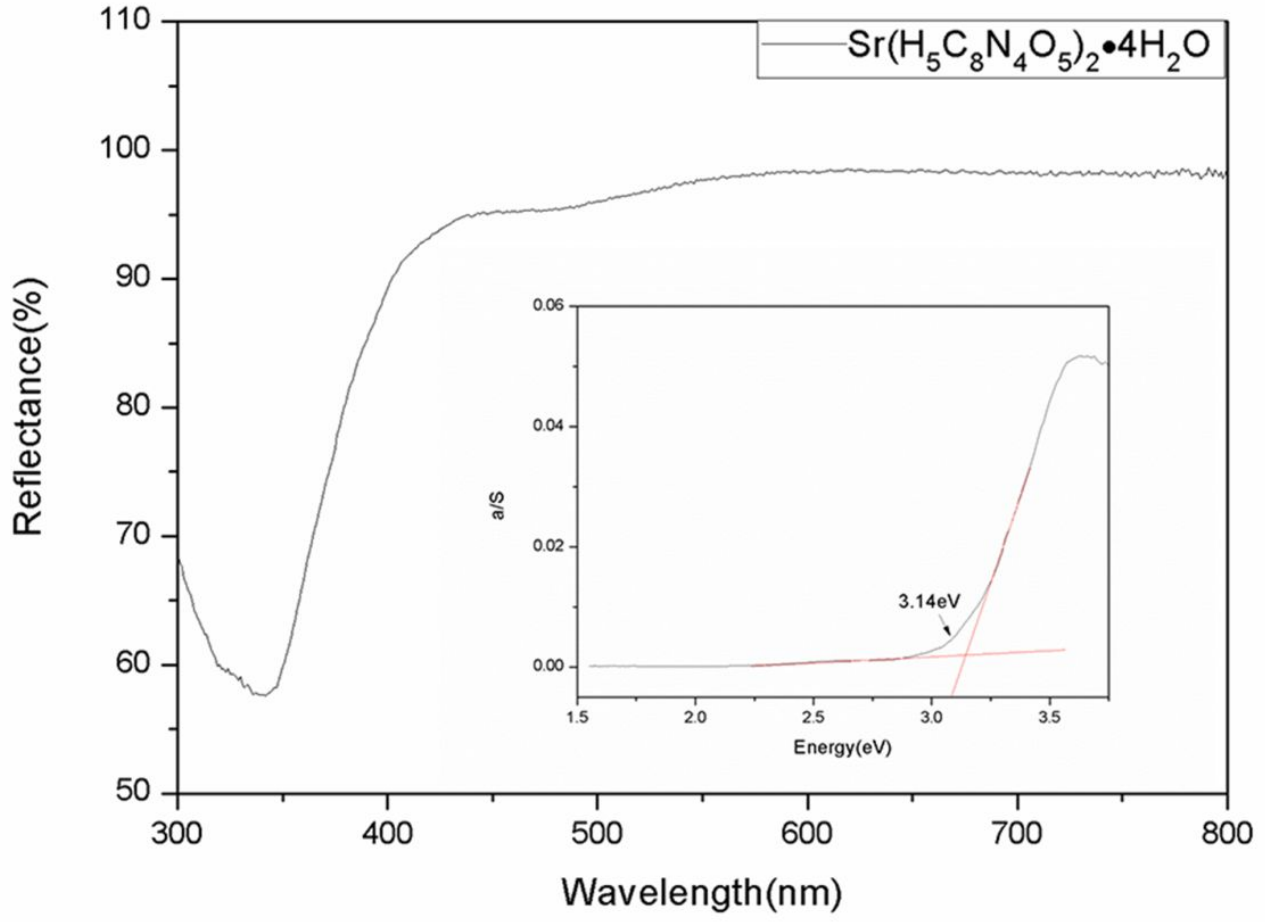

Figure S10. UV-vis-NIR diffuse reflectance spectrum of $\operatorname{Sr}\left(\mathrm{H}_{5} \mathrm{C}_{8} \mathrm{~N}_{4} \mathrm{O}_{5}\right)_{2} \cdot 4 \mathrm{H}_{2} \mathrm{O}$.

(a)

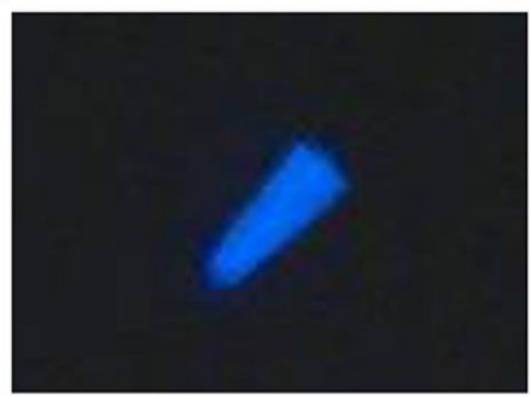

(c)

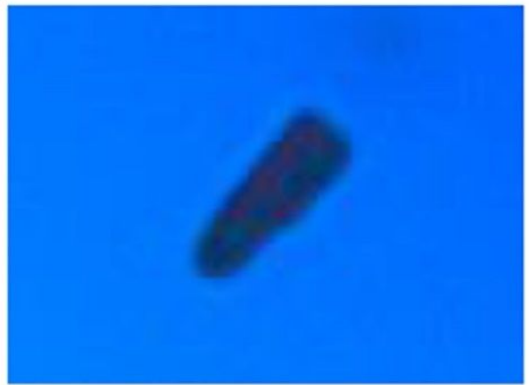

(b)

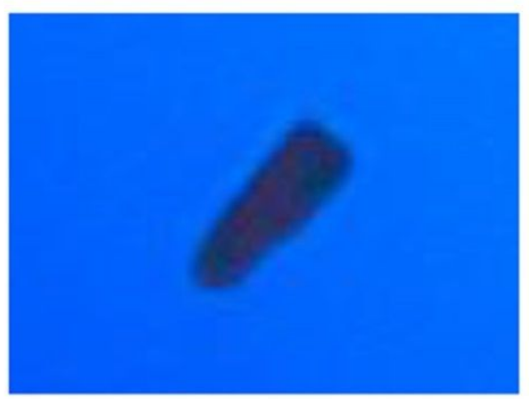

(d)

\section{$2.49 \mathrm{um}$}

Figure S11. (a)Photograph of $\mathrm{Ca}\left(\mathrm{H}_{3} \mathrm{C}_{4} \mathrm{~N}_{2} \mathrm{O}_{3}\right)_{2} \cdot \mathrm{H}_{2} \mathrm{O}$ for the measurement of birefringence, and (b) the positive and (c) negative rotation of compensatory, and (d) the thickness of measured $\mathrm{Ca}\left(\mathrm{H}_{3} \mathrm{C}_{4} \mathrm{~N}_{2} \mathrm{O}_{3}\right)_{2} \cdot \mathrm{H}_{2} \mathrm{O}$ crystal. 
(a)

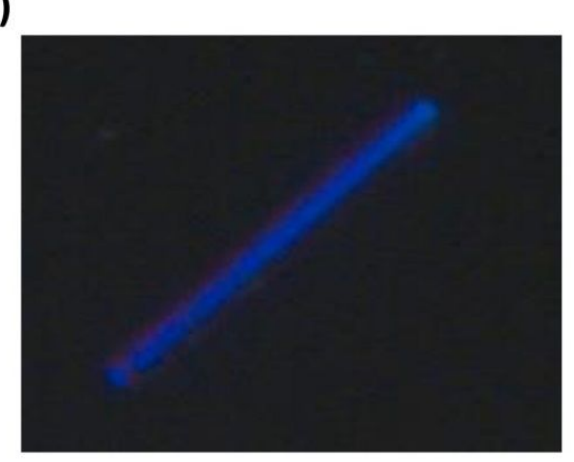

(c)

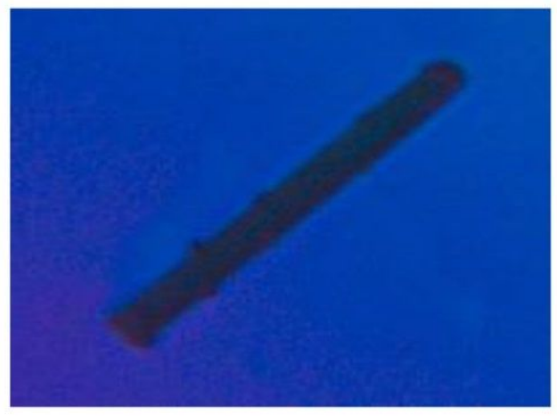

(b)

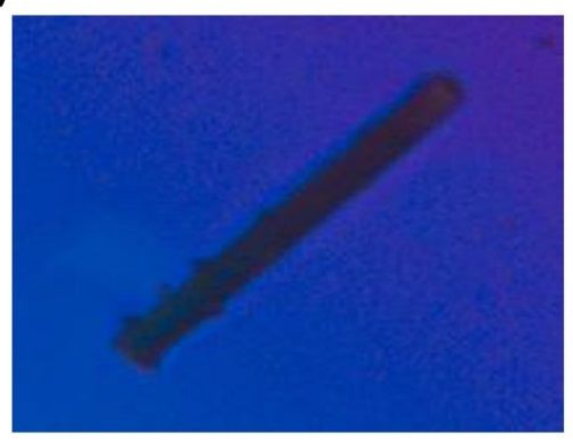

(d)

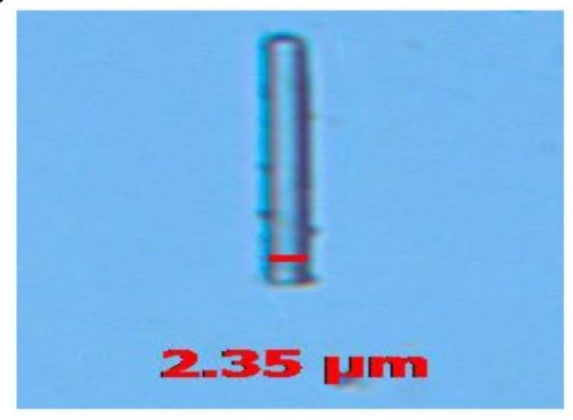

Figure S12. (a)Photograph of $\operatorname{Sr}\left(\mathrm{H}_{5} \mathrm{C}_{8} \mathrm{~N}_{4} \mathrm{O}_{5}\right)_{2} \cdot 4 \mathrm{H}_{2} \mathrm{O}$ for the measurement of birefringence, and (b) the positive and (c) negative rotation of compensatory, and (d) the thickness of measured $\mathrm{Sr}\left(\mathrm{H}_{5} \mathrm{C}_{8} \mathrm{~N}_{4} \mathrm{O}_{5}\right)_{2} \cdot 4 \mathrm{H}_{2} \mathrm{O}$ crystal.

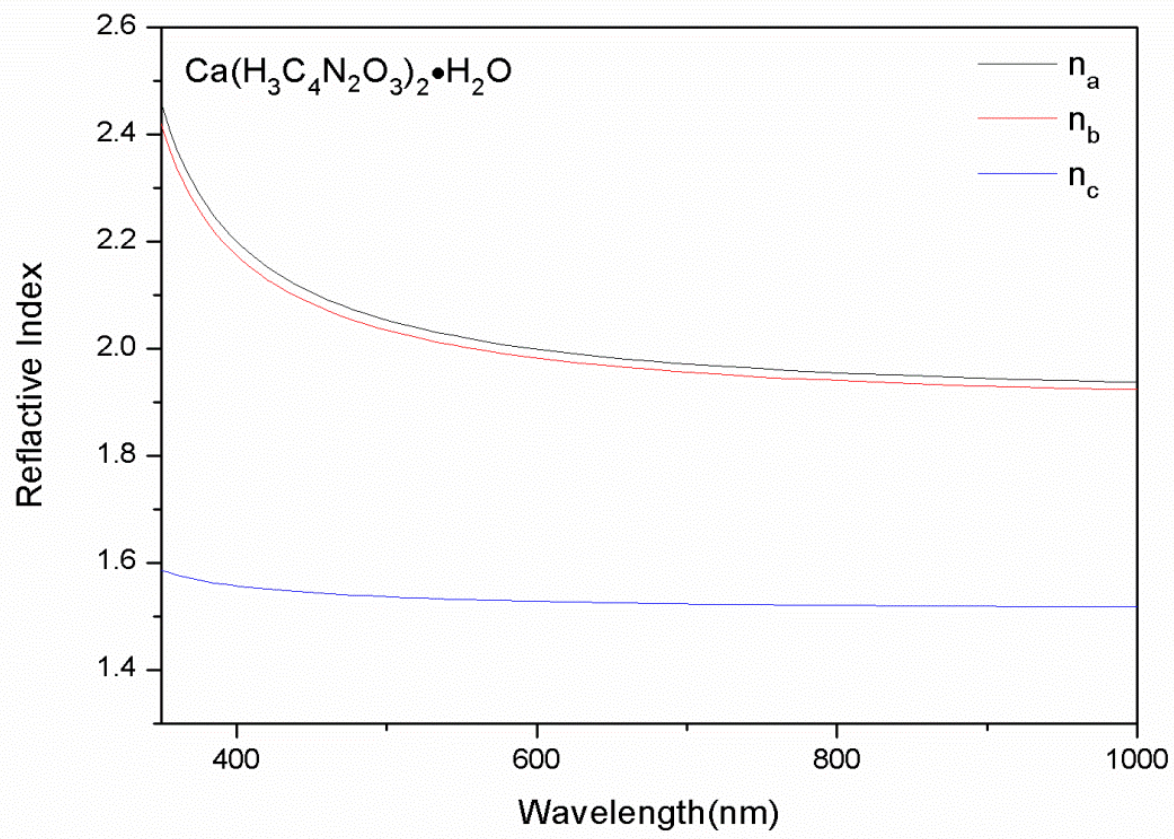

Figure S13. Calculated refractive indices of $\mathrm{Ca}\left(\mathrm{H}_{3} \mathrm{C}_{4} \mathrm{~N}_{2} \mathrm{O}_{3}\right)_{2} \cdot \mathrm{H}_{2} \mathrm{O}$. 


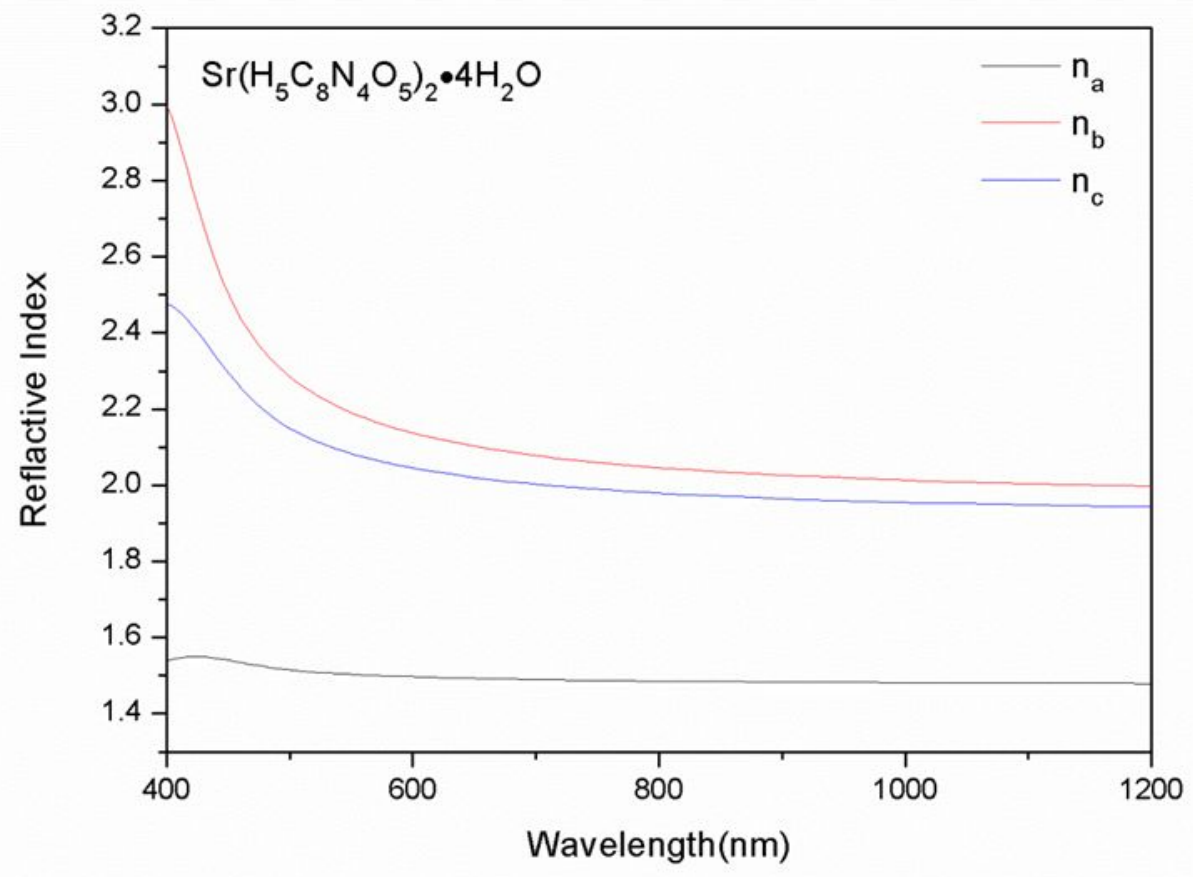

Figure S14. Calculated refractive indices of $\operatorname{Sr}\left(\mathrm{H}_{5} \mathrm{C}_{8} \mathrm{~N}_{4} \mathrm{O}_{5}\right)_{2} \cdot 4 \mathrm{H}_{2} \mathrm{O}$.

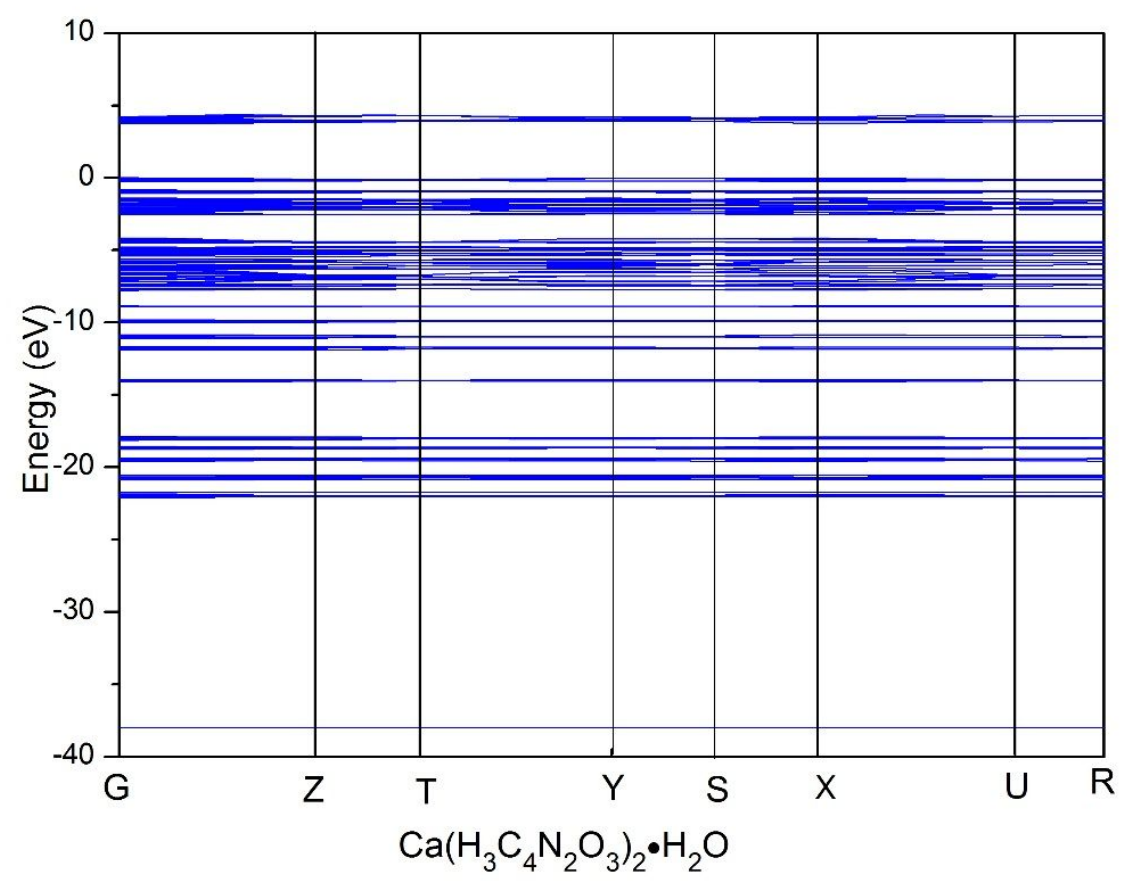

Figure S15. Band structures for $\mathrm{Ca}\left(\mathrm{H}_{3} \mathrm{C}_{4} \mathrm{~N}_{2} \mathrm{O}_{3}\right)_{2} \cdot \mathrm{H}_{2} \mathrm{O}$. 


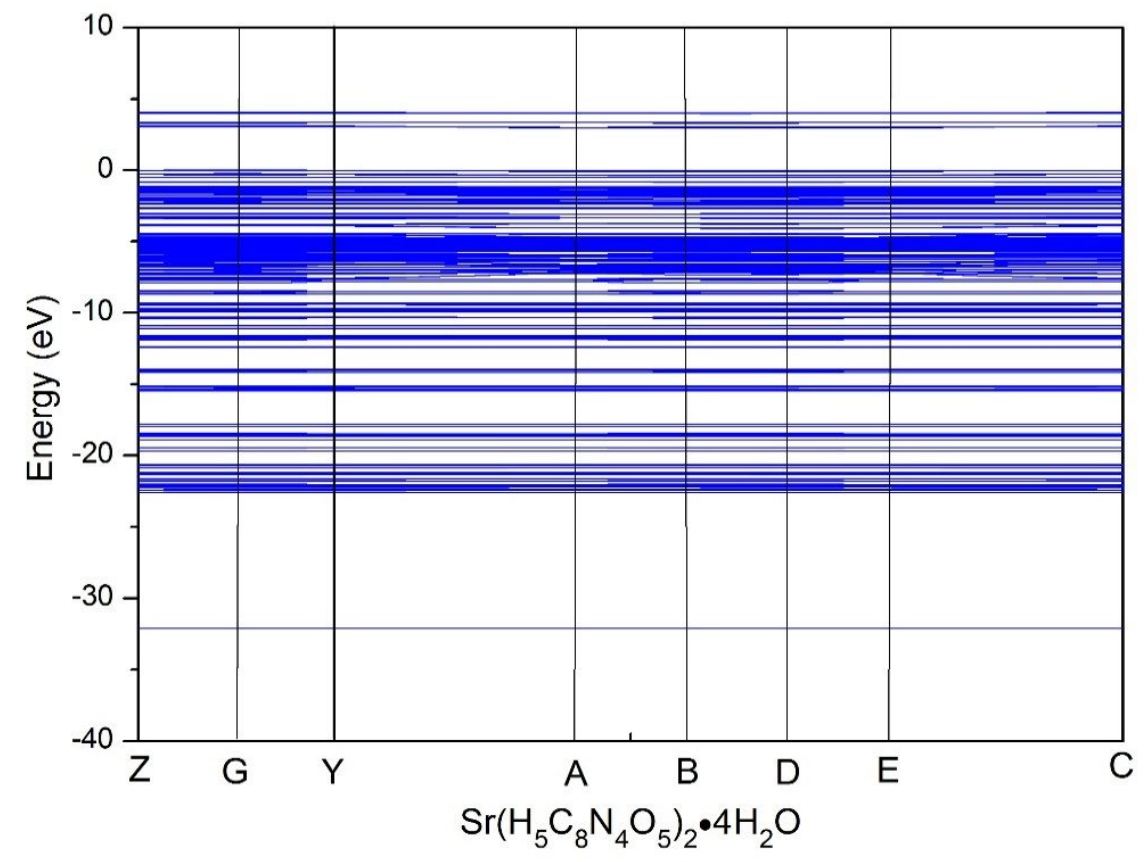

Figure S16. Band structures for $\mathrm{Sr}\left(\mathrm{H}_{5} \mathrm{C}_{8} \mathrm{~N}_{4} \mathrm{O}_{5}\right)_{2} \cdot 4 \mathrm{H}_{2} \mathrm{O}$.

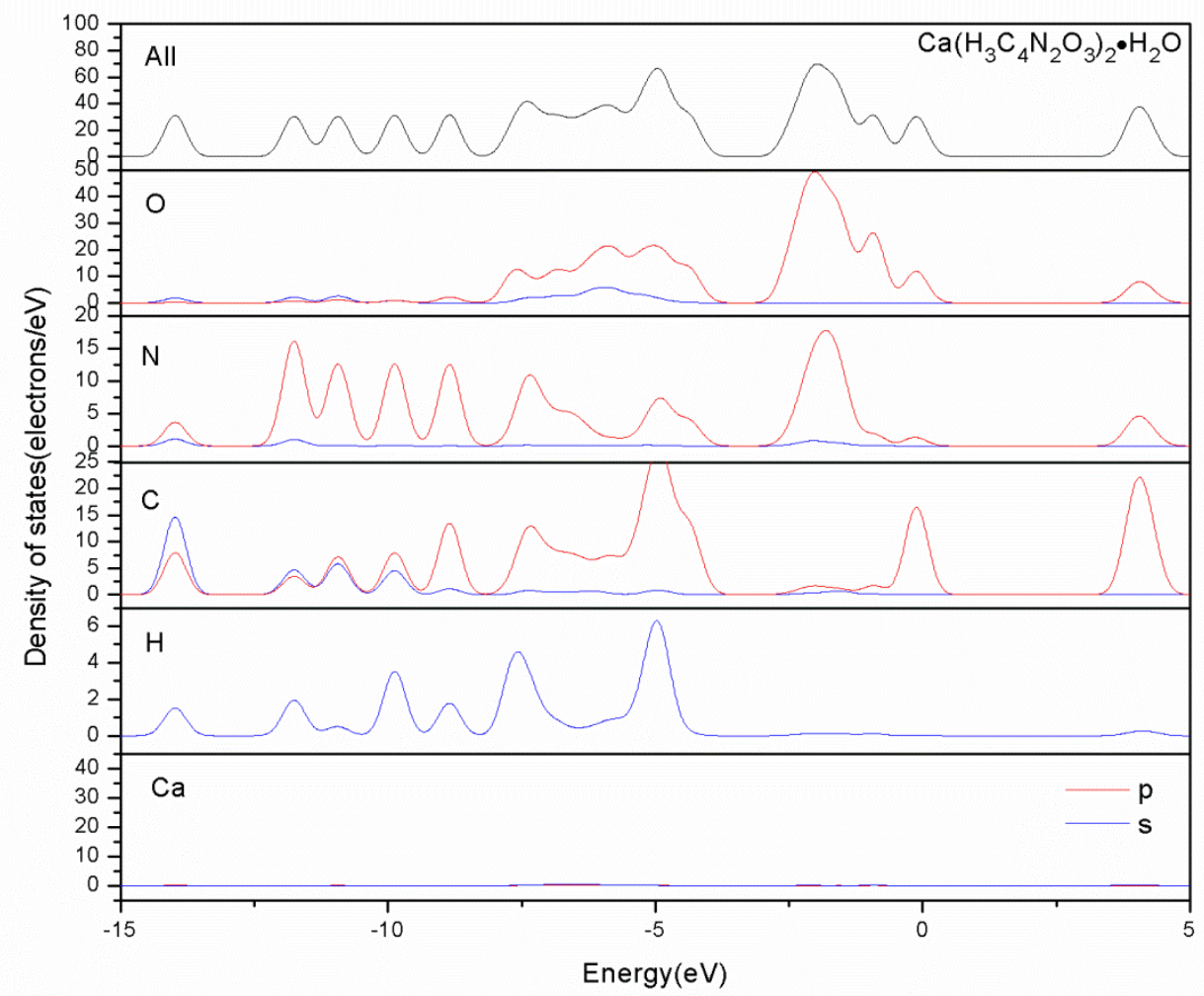

Figure S17. Densities of states for $\mathrm{Ca}\left(\mathrm{H}_{3} \mathrm{C}_{4} \mathrm{~N}_{2} \mathrm{O}_{3}\right)_{2} \cdot \mathrm{H}_{2} \mathrm{O}$. 


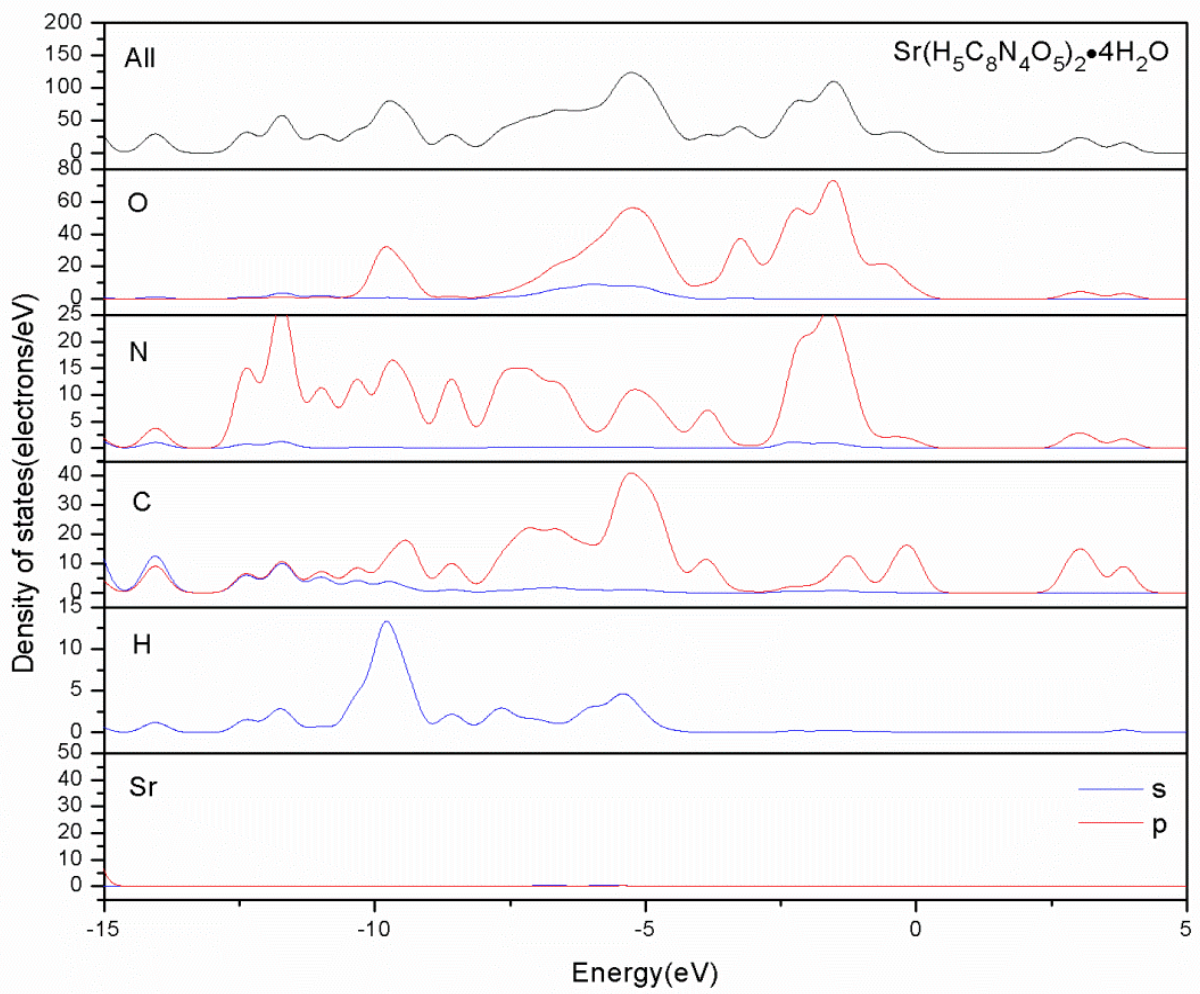

Figure S18. Densities of states for $\operatorname{Sr}\left(\mathrm{H}_{5} \mathrm{C}_{8} \mathrm{~N}_{4} \mathrm{O}_{5}\right)_{2} \cdot 4 \mathrm{H}_{2} \mathrm{O}$.

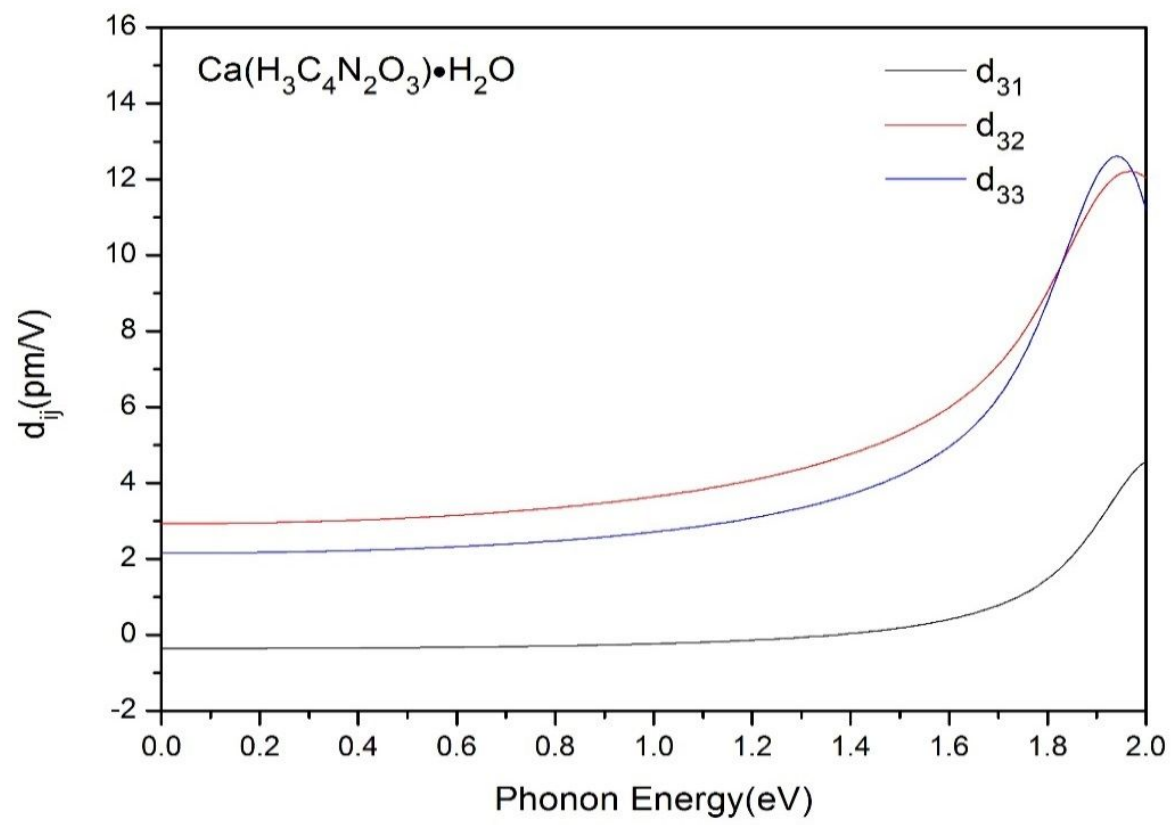

Figure S19. Calculated frequency-dependent second-harmonic generation coefficients for $\mathrm{Ca}\left(\mathrm{H}_{3} \mathrm{C}_{4} \mathrm{~N}_{2} \mathrm{O}_{3}\right)_{2} \cdot \mathrm{H}_{2} \mathrm{O}$. 
The Anionic Group Theory Calculation.

The macroscopic second-order susceptibility $\chi_{i j k}^{(2)}$ could be expressed by Eq. (1) according to the anionic group theory.

$$
\begin{array}{r}
\chi_{i j k}^{(2)}=\frac{F}{V} \sum_{a, b, c} g_{i a, j b, k c} \cdot \beta_{a b c}^{(2)}=\frac{F}{V} \cdot g_{i j k} \cdot \beta_{111}^{(2)} \\
\beta_{111}^{(2)}=-\beta_{122}^{(2)}=-\beta_{212}^{(2)}=-\beta_{221}^{(2)}(2)
\end{array}
$$

where $\mathrm{F}$ is the correction factor of the localized field, $\mathrm{V}$ is the volume of the unit cell, $\alpha_{\mathrm{ia}}, \alpha_{\mathrm{jb}}$, and $\alpha_{\mathrm{kc}}$ are the direction cosines between the macroscopic coordinate axes of the crystal and the microscopic coordinate axes of $\left(\mathrm{H}_{3} \mathrm{C}_{4} \mathrm{~N}_{2} \mathrm{O}_{3}\right)^{-}$groups, and $\beta$ is the microscopic secondorder susceptibility tensors of an individual group.

The geometrical factor g could be derived from Eq. (1).

$$
\mathrm{g}=\max \left(\mathrm{g}_{\mathrm{jik}}\right) ;(\mathrm{i}, \mathrm{j}, \mathrm{k}=1,2,3)(3)
$$

In case of unspontaneous polarization, the structural criterion $\mathrm{C}$ is defined as:

$$
C=\frac{g}{n}(4)
$$

where $\mathrm{n}$ is the number of anionic groups in a unit cell. 